\title{
The biomarkers of key miRNAs and target genes associated with acute myocardial infarction
}

\author{
Qi Wang ${ }^{1}$, Binyan Liu ${ }^{2,3}$, Yuanyong Wang ${ }^{4}$, Baochen Bai ${ }^{1}$, Tao Yu ${ }^{\text {Corresp., } 3}$, Ming Chu ${ }^{\text {Corresp. } 1,5}$ \\ 1 Department of Cardiology, The Affiliated hospital of Qingdao University, Qingdao, China \\ 2 School of Basic Medicine, Qingdao University, Qingdao, China \\ 3 Institute for translational medicine, Qingdao University, Qingdao, China \\ 4 Department of Thoracic Surgery, Affiliated Hospital of Qingdao University, Qingdao, China \\ 5 Department of Cardiology, The Affiliated Cardiovascular Hospital of Qingdao University, Qingdao, China \\ Corresponding Authors: Tao Yu, Ming Chu \\ Email address: yutao0112@qdu.edu.cn, chuxianmingqy@163.com
}

Background: Acute myocardial infarction (AMI) is considered one of the most prominent causes of death from cardiovascular disease worldwide. Knowledge of the molecular mechanisms underlying AMI remains limited. Accurate biomarkers are needed to predict the risk of AMI and would be beneficial for managing the incidence rate. The gold standard for the diagnosis of AMI, the cardiac troponin T (cTnT) assay, requires serial testing, and the timing of measurement with respect to symptoms affects the results. As attractive candidate diagnostic biomarkers in AMI, circulating microRNAs (miRNAs) are easily detectable, generally stable and tissue specific.

Methods: The Gene Expression Omnibus (GEO) database was used to compare miRNA expression between AMI and control samples, and the interactions between miRNAs and mRNAs were analysed for expression and function. Furthermore, a protein-protein interaction (PPI) network was constructed. The miRNAs identified in the bioinformatic analysis were verified by RT-qPCR in an H9C2 cell line. The miRNAs in plasma samples from patients with AMI $(n=11)$ and healthy controls $(n=11)$ were used to construct receiver operating characteristic (ROC) curves to evaluate the clinical prognostic value of the identified miRNAs.

Results: We identified 8 novel miRNAs as potential candidate diagnostic biomarkers for patients with AMI. In addition, the predicted target genes provide insight into the molecular mechanisms underlying AMI. 
1 The biomarkers of key miRNAs and target genes associated with acute myocardial 2 infarction

3

4 Qi Wang ${ }^{1}$, Bingyan Liu ${ }^{2,4}$, Yuanyong Wang ${ }^{5}$, Baochen Bai ${ }^{1}$, Tao Yu ${ }^{2 *}$, Xian-ming Chu ${ }^{1} 3^{*}$

5

$6{ }^{1}$ Department of Cardiology, The Affiliated hospital of Qingdao University, No. 59 Haier Road, 7 Qingdao 266100, China;

82 Institute for translational medicine, Qingdao University, No. 38 Dengzhou Road, 266021, 9 People's Republic of China;

$10{ }^{3}$ Department of Cardiology, The Affiliated Cardiovascular Hospital of Qingdao University, No. 115 Zhiquan Road, Qingdao 266000, China.

$12{ }^{4}$ School of Basic Medicine, Qingdao University, Qingdao, Shandong, China.

13 Department of Thoracic Surgery, Affiliated Hospital of Qingdao University

14

$15{ }^{*}$ Corresponding author:

16 Tao $\mathrm{Yu}^{2}, \mathrm{PhD}$

17

Center for Regenerative Medicine, Institute for translational medicine, Qingdao University, 38 Deng Zhou Road, Qingdao 266021, China

Tel: +86-532-82991791; fax: +86-532-82991791; e-mail: yutao0112@qdu.edu.cn

Xian-ming $\mathrm{Chu}^{1,3}, \mathrm{MD}$

1 Department of Cardiology, the Affiliated Hospital of Qingdao University, No. 59 Haier Road, Qingdao 266100, China; Tel.: +86-532-82913125;

2 The Affiliated Cardiovascular Hospital of Qingdao University, No. 5 Zhiquan Road, Qingdao 266000, China; Tel.: +86-532-66990020; fax: +86-532-66990020; e-mail: chuxianmingqy@163.com

2 


\section{Abstract}

36 Background: Acute myocardial infarction (AMI) is considered one of the most prominent causes of death from cardiovascular disease worldwide. Knowledge of the molecular mechanisms underlying AMI remains limited. Accurate biomarkers are needed to predict the risk of AMI and would be beneficial for managing the incidence rate. The gold standard for the diagnosis of AMI, the cardiac troponin $\mathrm{T}$ (cTnT) assay, requires serial testing, and the timing of measurement with respect to symptoms affects the results. As attractive candidate diagnostic biomarkers in AMI, circulating microRNAs (miRNAs) are easily detectable, generally stable and 43 tissue specific.

44 Methods: The Gene Expression Omnibus (GEO) database was used to compare miRNA expression between AMI and control samples, and the interactions between miRNAs and mRNAs were analysed for expression and function. Furthermore, a protein-protein interaction (PPI) network was constructed. The miRNAs identified in the bioinformatic analysis were verified by RT-qPCR in an H9C2 cell line. The miRNAs in plasma samples from patients with AMI $(n=11)$ and healthy controls $(n=11)$ were used to construct receiver operating characteristic (ROC) curves to evaluate the clinical prognostic value of the identified miRNAs.

51 Results: We identified 8 novel miRNAs as potential candidate diagnostic biomarkers for patients with AMI. In addition, the predicted target genes provide insight into the molecular mechanisms 53 underlying AMI.

54 Keywords: acute myocardial infarction, diagnostic biomarkers, miRNA-mRNA network, differentially expressed genes 


\section{Introduction}

Acute myocardial infarction (AMI) is the most common cardiac event worldwide and among cardiovascular diseases (CVDs) is a leading threat to human health (Guo et al. 2019). AMI is caused by acute coronary syndrome (ACS), which is induced by plaque ulceration or intravascular thrombosis and thrombotic material after rupture (Li et al. 2017). Early diagnosis and interventional therapy are important to minimize the damage to cardiac muscle and have the potential to significantly reduce mortality and improve prognosis (Braunwald 2012). Although the cardiac troponin T (cTnT) assay, the gold standard for diagnosis of AMI, has facilitated the diagnosis of AMI and contributed to lower mortality, it can lead to false positives in patients with chronic but stable coronary artery disease or healthy controls (Braunwald 2012). Therefore, novel biomarkers with high sensitivity and specificity are urgently needed to allow the early diagnosis of AMI and thereby improve clinical outcomes.

microRNAs (miRNAs), which are RNAs containing approximately 20 to 24 nucleotides, do not have the potential to encode proteins but can negatively regulate genes (Cheng et al. 2019). miRNAs restrain protein translation or mRNA degradation by binding to the 3'UTRs of messenger RNAs (mRNAs) (Fasanaro et al. 2010). Accumulating studies have revealed that miRNAs are involved in multifarious biological functions, including cell proliferation, apoptosis and inflammation, and exhibit strong correlations with mechanisms of disease, especially in cardiovascular disease (Feinberg \& Moore 2016). miRNAs have been identified as biomarkers of pathological events during the process of AMI (Boon \& Dimmeler 2015). In particular, the knockdown of miR-155 inhibits cardiomyocyte apoptosis in AMI-induced mice, and miR-155 is upregulated by negatively regulating the RNA-binding protein Quaking (QKI) (Guo \& Liu 2019). Cai et al. found that miR-29b-3p overexpression could protect cardiomyocytes against hypoxiainduced injury by negatively regulating the level of TRAF5, which suggests a potential therapeutic method for AMI (Cai \& Li 2019). Circulating miRNAs are easily detectable, relatively stable and tissue specific, making them attractive candidate biomarkers (Wang et al. 2010). Enhancing our understanding of the relationships between miRNAs and target genes can help reveal detailed mechanisms and identify novel biomarkers for AMI. In this study, we aimed to identify miRNAs with high clinical applicability for distinguishing patients with AMI from those without.

To that end, we identified circulating miRNAs that are differentially expressed (DE) in AMI by using integrated analysis. Gene expression profiles in AMI were acquired through the Gene Expression Omnibus (GEO) database. Then, a competitive endogenous RNA (ceRNA) network was constructed after a comprehensive analysis. Receiver operating characteristic (ROC) curve analysis was applied to analyse the diagnostic usefulness of the identified DE-miRNAs and genes. Finally, 8 potential miRNAs were identified as significant predictors of AMI. Our study may be helpful for elucidating the mechanisms of AMI pathogenesis and identifying diagnostic biomarkers for AMI.

\section{Materials and methods Subjects}


A total of 11 AMI patients and 11 healthy subjects were enrolled from Affiliated Hospital of Qingdao University between 2017 and 2018 (Supplemental Table 2). All of the AMI patients had been diagnosed for the first time and undergone a primary percutaneous coronary intervention (PCI). The diagnosis for AMI was made based on the following criteria: (i) acute ischaemic chest pain within $24 \mathrm{~h}$; (ii) electrocardiogram changes (pathological Q wave, ST-segment elevation or depression) and (iii) increases in cardiac biomarkers. The exclusion criteria were selected due to their potential influence on miRNA expression and were as follows: previous history of cardiac disease, tumour, renal insufficiency, surgery within the six previous months, and anticoagulant therapy. The study was conducted in accordance with the Declaration of Helsinki. The ethical committee of Affiliated Hospital of Qingdao University approved the study numbered QYFYWZLL25621.

\section{Sample collection and RNA isolation}

Blood samples were collected into EDTA tubes before coronary angiography and application of heparin. Serum was obtained after centrifugation at $3000 \mathrm{~g}$ for $10 \mathrm{~min}$ at $4^{\circ} \mathrm{C}$ to remove debris and stored in RNase-free tubes at $-80^{\circ} \mathrm{C}$ at Affiliated Hospital of Qingdao University until analysis. All participants were informed of the study details by the ethics committee of the hospital and provided informed consent. Total RNA from the serum samples was extracted using TRIzol reagent (Sigma, St. Louis, MO, USA) following the manufacturer's instructions. For normalization, 25 fmol Caenorhabditis elegans miR-39 (cel-miR-39) (Qiagen, Valencia, CA) was added to each serum sample after the addition of TRIzol, following previous methods.

\section{Data sources}

The data expression profiles of AMI were searched in the GEO database, and two independent datasets of research on AMI, GSE24591 and GSE31568, were included in our study. Using the genome-wide expression data of miRNAs obtained from the two selected independent cohorts, differential genes were screened according to the control group and AMI group of samples.

\section{Data preprocessing and identification of DEGs}

GEO2R, which is an interactive web tool that allows comparisons between two groups of samples to analyse almost any GEO series, was used to confirm DEGs between the control group and AMI group. The limma R package was applied by GEO2R and served as the processor to handle the supplied processed data tables. DEGs between the control and AMI groups were screened out according to the criteria $\mathrm{p}$ value less than .05 and absolute log fold change greater than 1 .

\section{Analyses of miRNA-mRNA targets}

Investigating the target genes of miRNAs is crucial for identifying the regulatory mechanisms and functions of miRNAs. Herein, we identified 8 DE-miRNAs and then predicted the targets of the DEGs by employing three miRNA-target tools: miRWalk V2.0 database, mirDIP, and miRTarBase. The miRNA targets were screened based on the overlapping results from the three 
websites. Then, the regulatory networks of the miRNA-mRNA pairs were extracted (based on an expression fold change $>2.5$ and an FDR $<0.05$ ) and visualized using Cytoscape software (Smoot et al. 2011).

\section{Gene Ontology (GO) annotation and Kyoto Encyclopaedia of Genes and Genomes (KEGG) pathway enrichment analyses of the DEGs}

GO annotation and KEGG pathway enrichment analyses of the DEGs were performed using the Database for Annotation, Visualization and Integrated Discovery (DAVID) (selected with enrichment significance evaluated at $\mathrm{p}<.05$ ), which revealed the biological processes (BPs), cellular components (CCs), molecular functions (MFs) and pathways associated with the DEmiRNAs.

\section{Protein-protein interaction (PPI) network construction and hub gene identification}

To gain insights into the interactions of the 591 target genes of the identified miRNAs, a PPI network was constructed and analysed with the STRING tool to reveal the molecular mechanisms underlying AMI. Target genes in the PPI network serve as nodes, the lines between two nodes denote associated interactions, and the strengthof the interaction is expressed by the colour of the line. The hub genes, which were defined as genes that play essential roles in the network, were distinguished according to the cutoff criteria of degree calculated by cytoHubba in Cytoscape. The corresponding interactions were visualized using Cytoscape software (http://cytoscape.org/) (Su et al. 2014).

\section{Cell culture and treatment}

The H9C2 cell line was obtained from the Shanghai Institutes for Biological Sciences (Shanghai, China) and cultured in Dulbecco's modified Eagle's medium (DMEM) containing 10\% FBS (ExCell Bio, Shanghai, China) and 1\% antibiotics. The cells were cultivated in a humidified atmosphere with $5 \% \mathrm{CO} 2$ at $37^{\circ} \mathrm{C}$. The cells were trypsinised to generate single cell suspensions at $80 \%$ confluency. The cells were treated with $2 \mu \mathrm{M}$ doxorubicin (DOX) for $24 \mathrm{~h}$.

RT-qPCR analysis

A reverse transcription kit (Takara, Otsu, Japan) was applied to synthesize the cDNA. RT-qPCR was accomplished using SYBR Green PCR Master Mix (Yeasen, Shanghai, China). The forward primers arepresented in Table 1, and the same reverse primer with the sequence 5' GTGCAGGGTCCGAGGT-3' was used for all miRNAs.

The average expression levels of serum miRNAs were normalized against cel-miR-39 (Qiagen, Valencia, CA), and the expression of cell-derived miRNAs were normalized against U6 (Takara, Otsu, Japan).

Fold changes in miRNA expression were calculated using the $2^{-\Delta \Delta \mathrm{Ct}}$ method for each sample in triplicate (Chang et al. 2009). Taking the calculation method of miRNA expression in plasma as an example, $\Delta \Delta \mathrm{Ct}=\left[\left(\mathrm{Ct}{ }_{\text {miRNA }}-\mathrm{Ct}\right.\right.$ cel-miRNA-39 $)$ diseased - $(\mathrm{Ct}$ miRNA- $\mathrm{Ct}$ cel-miRNA-39) control $]$. In brief, with this method, the $\mathrm{Ct}$ values from the target miRNA in both AMI and control group are 
180

adjusted in relation to the $\mathrm{Ct}$ of a normalizer RNA (cel-miR-39), which resulted in $\Delta \mathrm{Ct}$. In order to compare diseased and control samples, we calculated $\Delta \Delta \mathrm{Ct}$ values, which allowed us to determine the magnitude of the difference in miRNA expression. To ensure consistent measurements throughout all assays, for each PCR amplification reaction, three independent RNA samples were loaded as internal controls.

\section{ROC curves}

ROC curves were constructed to discriminate AMI patients from control subjects for the plasma miRNAs, and the areas under the ROC curves (AUCs) were analysed to assess the diagnostic accuracy of each identified miRNA. Herein, a normalized miRNA score was used to represent the expression level of the selected miRNA in the AMI group relative to that in the control group (Goren et al. 2012). In brief, we used miRNA scores, which were calculated by subtracting the normalized $\mathrm{Ct}$ from 40 and then adjusted by deducting the minimal score, leading to miRNA scores with a lower bound of 0. All statistical analyses were performed using SPSS 13.0 (Chicago, IL, USA).

Statistical analyses

Student's t-test was carried out using GraphPad Prism 5 to compare test and control samples. For the analysis of clinical characteristics in AMI patients and control individuals, data were presented as means \pm standard deviations (SD) for quantitative variables. Mean values of quantitative variables were evaluated by Student's t-test, or Mann-Whitney U-test when Student's t-test were not satisfied. $\mathrm{p}<.05$ was considered to indicate a statistically significant difference. All statistical analyses were performed using SPSS 13.0 (Chicago, IL, USA).

\section{Results}

\section{Identification of DE-miRNAs}

$|\log 2 \mathrm{FC}|>1$ and $\mathrm{p}$ value $<.05$ were considered as criteria to screen the DE-miRNAs. Among the selected GEO datasets, 27 DE-miRNAs, including 25 downregulated and 2 upregulated genes, were found in the GSE24591 profile (Figure 1A), whereas 307 DE-miRNAs, including 132 upregulated and 175 downregulated genes, were found in the GSE31568 profile (Figure 1B). The candidate DE-miRNAs generated by the two datasets were intersected using a Venn diagram (Figure 1C). All intersecting DE-miRNAs are shown in Table 2 and included hsa-miR-545, hsamiR-139-3p, hsa-miR-101, hsa-miR-24-1, hsa-miR-598, hsa-miR-33a, hsa-miR-142-3p, and hsamiR-34a.

\section{miRNA-target gene interactions}

Following data preprocessing and analysis of the three databases, an overlap of 591 gene pairs from 8 DE-miRNAs was obtained among the databases. These overlapping pairs were used to predict the target genes that interact with the miRNAs. The predictions were verified by more than four algorithms, including miRDB, RNA22, RNAhybrid, and TargetScan. The network of 
220

221

222

223

224

225

226

227

228

229

230

231

232

233

234

235

236

237

238

239

240

241

242

243

244

245

246

247

248

249

250

251

252

253

254

255

256

257

258

259

260

miRNA-mRNA interactions was visualized in Cytoscape, as shown in Figure 2, and the target genes are listed in Table 3.

\section{Enrichment analyses of the target genes}

To investigate the functions of the target genes, GO annotation and KEGG pathway analyses of the interacting 591 genes from GSE24591 and GSE31568 were performed utilizing the DAVID online tool. The top $10 \mathrm{GO}$ and KEGG items, including the BPs, CCs, MFs and KEGG pathways that were significantly enriched, are listed in Figure 3A-D. The significantly enriched entries for BPs were positive regulation of transcription from the RNA polymerase II promoter, transcription, and DNA-templated and negative regulation of transcription from the RNA polymerase II promoter (Figure 3A). Furthermore, the nucleus, cytoplasm, and nucleoplasm accounted for the majority of the CC terms (Figure 3B). The most enriched MFs were functions in metal ion binding, zinc ion binding and poly (A) RNA binding (Figure 3C). In the MF category, the top 10 most highly regulated DE-miRNAs were significantly enriched in the pathways of cancer and the PI3K-Akt pathway. Intriguingly, the enrichment in adrenergic signalling in cardiomyocytes was found to be closely related to AMI (Figure 3D).

\section{PPI network}

To distinguish the connections among the 591 target genes, we mapped the PPIs using the logical data originating from the STRING database (http://strin g.embl.de/). With degree as the criterion, the top 100 linked DE-miRNAs were identified, as shown in Figure 4. The network is composed of 100 nodes and 700 edges and has an average local clustering coefficient of .467. The top 10 genes with a high-ranking degree are labelled in purple and associated with much larger circles; all the edges are distinguished based on connection score (Figure 4).

\section{Biological analysis of the hub genes}

Highly connected proteins in a network are master keys of regulation and are defined as hub proteins ( $\mathrm{Yu}$ et al. 2017). The hub proteins in the present study included CTNNB1, CCND1, NOTCH1, EZH2, MTOR, BTRC, RAC1, CDKN1A, CDKN1B, and MAP2K1. They were identified by evaluating degree with the Biological Networks Gene Ontology tool (BiNGO) plugin of Cytoscape, which considered the top ten closely related interactions (Table 4); these involved 10 nodes and 35 edges (Figure 5A). Additionally, KEGG analysis was performed on the potential hub genes (Figure 5B), and the top 10 enrichment pathways were identified (Figure $5 \mathrm{C})$.

\section{Validation of the identified miRNAs}

The expression levels of the identified miRNAs were quantified by RT-qPCR in H9C2 cells treated with DOX to verify the results of the bioinformatic analyses. Emerging studies have illuminated the role of cardiomyocyte apoptosis in DOX-induced myocardial damage, which is similar to the proceeding of AMI (Catanzaro et al. 2019). Based on our previous study, we treated H9C2 cells with $2 \mu \mathrm{M}$ DOX. As shown in Figure 6, miR-34a, miR-101 and miR-598 
261 were upregulated, and miR-24-1, miR-33a, miR-139-3p, miR-142-3p, and miR-545 were 262 downregulated. Furthermore, the results of miR-24-1*, miR-33a, miR-34a, miR-101, miR-139$2633 \mathrm{p}$, and miR-545 in patients were consistent with the results obtained for the tissue cultures. 264 However, the expression of miR-142-3p did not have significant change in blood samples and 265 miR-598 was upregulated in blood but decreases in tissues (Supplement Figure 1). To investigate 266 the efficacy of DE-miRNAs as potential biomarkers of AMI, we performed ROC curve analysis 267 of patients with AMI and patients without AMI. The expression levels of the DE-miRNAs were

268

269

270

271

272

273

274

275

276

277

278

279

280

281

282

283

284

285

286

287

288

289

290

291

292

293

294

295

296

297

298

299

300

301 significantly different between AMI patients and control individuals (Figure 7). AUC values were used to evaluate the potential of the DE-miRNAs as diagnostic markers. The AUC values of miR-24-1 and miR-545 were greater than .9, and these DE-miRNAs also had the highest accuracies. Moreover, all five miRNAs had high specificity with AUCs> 7 except for miR-142$3 p$ that the accuracy is likely to take place when the AUC above 7 (Catanzaro et al. 2019). These results indicated that the predicted miRNAs, especially miR-24-1 and miR-545, have potential for clinical application.

Relationships to conventional prognostic markers

To further evaluate the potential of circulating miRNAs as cardiac biomarkers, we tested whether the levels of identified miRNAs correlate with troponin $\mathrm{T}$ (TnT) level. miR-24-1 and miR-545 were strongly correlated with TnT $\left(\mathrm{r}=-0.722, \mathrm{p}<1 * 10^{-3}\right.$ and $\left.\mathrm{r}=-0.57, \mathrm{p}=0.006\right)$. miR-101, miR-139-3p, and miR-598 remained correlated with TnT levels in AMI patients $(\mathrm{r}=-0.444, \mathrm{p}=$ 0.038 for miR-101, $r=-0.425, p=0.048$ for miR-139-3p and $r=-0.425, p=0.048$ for miR598). However, miR-33a, miR-34a, and miR-142-3p was not correlated with TnT which showed in Supplement table 3. Combining ROC analysis results, we concluded that miR-24-1 might be the most potential biomarker in AMI.

\section{Discussion}

AMI, commonly referred to as acute heart attack, is generally acknowledged as the outcome of sudden ischaemia that results in insufficient blood supply and a subsequent imbalance between the supply and demand of oxygen induced by cardiomyocyte death (Vogel et al. 2019). AMI is a central contributor to the global disease burden, occurring in $4-10 \%$ of people under 45 years, with a massive number of patients still suffering recurrent cardiovascular events after treatment with medication or primary PCI (Tan et al. 2016). Previous studies have identified potential mechanisms and biomarkers for early diagnosis and treatment. Cardiac troponin (cTn) has served as the gold standard for AMI diagnosis and is routinely applied for patients with suspected ACS to rule-in or rule-out AMI (Sandoval et al. 2017). Nevertheless, with the advancing sensitivity of cTn assay, the assay has exceeded the ninety-ninth percentile for stable chronic conditions, weakening its specificity for the diagnosis of AMI (Park et al. 2017). This observation demonstrates that there is an urgent need for the identification of novel diagnostic markers and therapeutic targets with minimal risk of adverse effects and maximum sensitivity and specificity (Cruz et al. 2019). Various investigations have revealed that miRNAs can potentially predict CVDs by modulating the ceRNA network, thus providing a therapeutic option, especially in 
302

303

304

305

306

307

308

309

310

311

312

313

314

315

316

317

318

319

320

321

322

323

324

325

326

327

328

329

330

331

332

333

334

335

336

337

338

339

340

341

342

AMI (Lucas et al. 2018). The downregulation of miR-155 expression restrains apoptosis and maintains a proliferative effect in cardiomyocytes by targeting QKI and can thereby serve as a therapeutic marker for MI. Accordingly, strategies for the diagnosis and treatment of AMI could be furnished by analysing correlative data in the GEO database (Cruz et al. 2019) and generating an AMI-associated miRNA-mRNA regulatory network for clinical applications regarding diagnosis, therapy, and prognosis.

In this study, 27 DEGs in the GSE24591 dataset and 307 DEGs in the GSE31568 dataset were screened in AMI and control blood samples based on the differential analysis of GEO2R in the GEO database. Furthermore, 8 collective miRNAs (miR-545, miR-139-3p, miR-101, miR-24-1, miR-598, miR-33a, miR-142-3p, and miR-34a) were selected and identified as DE in AMI; there were few common DEGs because the two datasets were independent. An interaction network of miRNAs and mRNAs was constructed by using three websites, miRWalk V2.0, mirDIP, and miRTarBase, and more than 4 online prediction tools, including miRDB, RNA22, RNAhybrid, and TargetScan. In addition, GO and KEGG enrichment analyses of the mRNAs in the ceRNA network were performed. The PPI network, analysed via the STRING database and visualized by Cytoscape software, showed that 591 target proteins and 10 hub genes were significantly closely associated with the miRNAs. The expression validation and ROC analyses of these DE-miRNAs based on the RT-qPCR data supported the above results, which could quantify the diagnostic availability of the identified DE-miRNAs.

Moreover, among the 8 miRNAs that might exert effects on the development of AMI, miR34a has been identified in mechanistic studies as a biomarker for AMI. It is highly expressed in adult mice after MI and associated with a thin wall of the left ventricle (LV) (Qipshidze Kelm et al. 2018). Yang et al. found that the suppression of miR-34a facilitated cardiac function following MI partly by modulating the interrelated genes involved in cell proliferation and the cell cycle, including Bcl2, Cyclin D1, and Sirt1, which revealed the potential of miR-34a to boost endogenous repair/regeneration in the adult heart (Yang et al. 2015). Additionally, the remaining 7 miRNAs have been shown to regulate cardiac performance. miR-545, the negatively correlated target of HOTAIR, promotes cell apoptosis through the HOTAIR/miR545/EGFR/MAPK axis (Li et al. 2018). miR-101 has been shown to mitigate the deterioration of cardiac function in post-MI rats (Pan et al. 2012), and it can protect cardiac fibroblasts from hypoxia-induced apoptosis by restraining the TGF- $\beta$ signalling pathway, as shown by Zhao et al. (Zhao et al. 2015). In contrast, miR-33 deteriorates myocardial fibrosis via the inhibition of MMP16 and the stimulation of p38 MAPK signalling (Chen et al. 2018). After $I / R$, the expression of miR-139-3p increases and is downregulated by Urocortin 1 (Ucn-1), and the overexpression of miR-139-3p promotes the expression of genes involved in cell death and apoptosis (Díaz et al. 2017). miR-24-1 was found to be significantly hypermethylated in ischaemic cardiomyopathy (ISCM) and dilated cardiomyopathy (DCM) and significantly reduced in an ISCM group (Glezeva et al. 2019). Additionally, miR-598 was identified as a significant predictor of heart failure (HF) in a dyspnoea cohort (Ellis et al. 2013), and miR-142$3 p$ sponged by lncRNA TUG1 has been suggested to potentially alleviate myocardial injury ( $\mathrm{Su}$ et al. 2019). Thus, more mechanistic research is needed to explore the potential functions of the 
343

344

345

346

347

348

349

350

351

352

353

354

355

356

357

358

359

360

361

362

363

364

365

366

367

368

369

370

371

372

373

374

375

376

377

378

379

380

381

382

383

identified miRNAs in AMI. We found that several hub genes and correlative mechanism pathways including Notch1, CTNNB1, RAC1, and MTOR had greater diagnostic potential for AMI. The Notch1 activation pathway manages cardiac AMPK signalling by interacting with LKB1 during myocardial infarction (Yang et al. 2016). Spermidine (SPD) has been suggested to be involved in the cardiac dysfunction induced by MI by promoting autophagy in the AMPK/mTOR pathway (Yan et al. 2019). The inhibition of Annexin A3 (ANXA3) has been reported to accelerate cardiomyocyte maintenance by activating PI3K/Akt signalling in rats with AMI (Meng et al. 2019). RAC1 has been shown to inhibit the death of cardiac myocytes stimulated by hypoxia and modify the phosphorylation levels of PI3K, AKT, MAPK and ERK, which are significant factors of MI (Wang et al. 2017). Overall, we inferred that the 4 hub genes might be regarded as diagnostic biomarkers and recovery monitors in AMI.

There are several limitations to our present study. The number of samples we obtained from GSE24591 and GSE31568 was small, generating some bias when analysing the DE-miRNAs, and more blood samples are needed for validation with RT-qPCR in further research. In addition, the functions and molecular mechanisms of genes are very complicated, and predictions based only on bioinformatics need cellular and animal experiments for verification.

\section{Conclusions}

Based on GEO database analysis, bioinformatic analysis, and experimental verification, we not only identified 8 significant DE-miRNAs in AMI but also detected 10 hub genes that may serve as potential biomarkers of AMI. Our findings might provide reliable candidate biomarkers for the precise diagnosis and individualized treatment of AMI and the development of further clinical applications in AMI.

\section{Acknowledgements}

This work was supported by the National Natural Science Foundation of China (No. 31701208, 81870331).

\section{Conflict of interest}

The authors have declared that no competing interest exists.

\section{Authors' contributions}

Qi Wang conceived, designed and performed the experiments, contributed reagents/materials/analysis tools, and prepared figures and/or tables. Bingyan Liu contributed reagents/materials/analysis tools and prepared figures and/or tables. Yuanyong Wang analysed the data and prepared figures and/or tables. Baochen Bai analysed the data, prepared figures and/or tables, and approved the final draft. Tao Yu and Xian-ming Chu conceived and designed the experiments, authored or reviewed drafts of the paper, and approved the final draft. All authors read and approved the final manuscript. 
384

385

386

387

388

389

390

391

392

393

394

395

396

397

398

399

400

401

402

403

404

405

406

407

408

409

410

411

412

413

414

415

416

417

418

419

420

421

422

423

424

\section{References}

Boon RA, and Dimmeler S. 2015. MicroRNAs in myocardial infarction. Nature Reviews: Cardiology 12:135-142. 10.1038/nrcardio.2014.207

Braunwald E. 2012. Unstable angina and non-ST elevation myocardial infarction. American Journal of Respiratory and Critical Care Medicine 185:924-932. 10.1164/rccm.201109$1745 \mathrm{CI}$ 
Cai Y, and Li Y. 2019. Upregulation of miR-29b-3p protects cardiomyocytes from hypoxiainduced apoptosis by targeting TRAF5. Cellular \& Molecular Biology Letters 24:27. 10.1186/s11658-019-0151-3

Catanzaro MP, Weiner A, Kaminaris A, Li C, Cai F, Zhao F, Kobayashi S, Kobayashi T, Huang Y, Sesaki H, and Liang Q. 2019. Doxorubicin-induced cardiomyocyte death is mediated by unchecked mitochondrial fission and mitophagy. FASEB Journal:fj201802663R. 10.1096/fj.201802663R

Chang S, Chen W, and Yang J. 2009. Another formula for calculating the gene change rate in real-time RT-PCR. Molecular Biology Reports 36:2165-2168. 10.1007/s11033-0089430-1

Chen Z, Ding HS, Guo X, Shen JJ, Fan D, Huang Y, and Huang CX. 2018. MiR-33 promotes myocardial fibrosis by inhibiting MMP16 and stimulating p38 MAPK signaling. Oncotarget 9:22047-22057. 10.18632/oncotarget.25173

Cheng M, Yang J, Zhao X, Zhang E, Zeng Q, Yu Y, Yang L, Wu B, Yi G, Mao X, Huang K, Dong N, Xie M, Limdi NA, Prabhu SD, Zhang J, and Qin G. 2019. Circulating myocardial microRNAs from infarcted hearts are carried in exosomes and mobilise bone marrow progenitor cells. Nat Commun 10:959. 10.1038/s41467-019-08895-7

Cruz MS, da Silva AMG, de Souza KSC, Luchessi AD, and Silbiger VN. 2019. miRNAs emerge as circulating biomarkers of post-myocardial infarction heart failure. Heart Failure Reviews. 10.1007/s10741-019-09821-1

Díaz I, Calderón-Sánchez E, Toro RD, Ávila-Médina J, de Rojas-de Pedro ES, DomínguezRodríguez A, Rosado JA, Hmadcha A, Ordóñez A, and Smani T. 2017. miR-125a, miR139 and miR-324 contribute to Urocortin protection against myocardial ischemiareperfusion injury. Scientific Reports 7. 10.1038/s41598-017-09198-X

Ellis KL, Cameron VA, Troughton RW, Frampton CM, Ellmers LJ, and Richards AM. 2013. Circulating microRNAs as candidate markers to distinguish heart failure in breathless patients. European Journal of Heart Failure 15:1138-1147. 10.1093/eurjhf/hft078

Fasanaro P, Greco S, Ivan M, Capogrossi MC, and Martelli F. 2010. microRNA: emerging therapeutic targets in acute ischemic diseases. Pharmacology and Therapeutics 125:92104. 10.1016/j.pharmthera.2009.10.003

Feinberg MW, and Moore KJ. 2016. MicroRNA Regulation of Atherosclerosis. Circulation Research 118:703-720. 10.1161/circresaha.115.306300

Glezeva N, Moran B, Collier P, Moravec CS, Phelan D, Donnellan E, Russell-Hallinan A, O'Connor DP, Gallagher WM, Gallagher J, McDonald K, Ledwidge M, Baugh J, Das S, and Watson CJ. 2019. Targeted DNA Methylation Profiling of Human Cardiac Tissue Reveals Novel Epigenetic Traits and Gene Deregulation Across Different Heart Failure Patient Subtypes. Circulation: Heart Failure 12:e005765. 10.1161/CIRCHEARTFAILURE.118.005765

Goren Y, Kushnir M, Zafrir B, Tabak S, Lewis BS, and Amir O. 2012. Serum levels of microRNAs in patients with heart failure. European Journal of Heart Failure 14:147-154. 10.1093/eurjhf/hfr155 
466

467

468

469

470

471

472

473

474

475

476

477

478

479

480

481

482

483

484

485

486

487

488

489

490

491

492

493

494

495

496

497

498

499

500

501

502

503

504

505

506

Guo J, and Liu HB. 2019. MicroRNA-155 Promotes Myocardial Infarction-Induced Apoptosis by Targeting RNA-Binding Protein QKI. 2019:4579806. 10.1155/2019/4579806

Guo J, Liu HB, Sun C, Yan XQ, Hu J, Yu J, Yuan Y, and Du ZM. 2019. MicroRNA-155 Promotes Myocardial Infarction-Induced Apoptosis by Targeting RNA-Binding Protein QKI. Oxidative Medicine and Cellular Longevity 2019:4579806. 10.1155/2019/4579806

Li X, Zhou J, and Huang K. 2017. Inhibition of the lncRNA Mirt1 Attenuates Acute Myocardial Infarction by Suppressing NF-kappaB Activation. Cellular Physiology and Biochemistry 42:1153-1164. 10.1159/000478870

Li Y, Zhao W, Shi R, Jia J, Li X, and Cheng J. 2018. Rs4759314 polymorphism located in HOTAIR is associated with the risk of congenital heart disease by alternating downstream signaling via reducing its expression. Journal of Cellular Biochemistry 119:8112-8122. 10.1002/jcb.26736

Lucas T, Bonauer A, and Dimmeler S. 2018. RNA Therapeutics in Cardiovascular Disease. Circulation Research 123:205-220. 10.1161/CIRCRESAHA.117.311311

Meng H, Zhang Y, An ST, and Chen Y. 2019. Annexin A3 gene silencing promotes myocardial cell repair through activation of the PI3K/Akt signaling pathway in rats with acute myocardial infarction. Journal of Cellular Physiology 234:10535-10546. 10.1002/jcp. 27717

Pan Z, Sun X, Shan H, Wang N, Wang J, Ren J, Feng S, Xie L, Lu C, Yuan Y, Zhang Y, Wang Y, Lu Y, and Yang B. 2012. MicroRNA-101 inhibited postinfarct cardiac fibrosis and improved left ventricular compliance via the FBJ osteosarcoma oncogene/transforming growth factor-betal pathway. Circulation 126:840-850. 10.1161/CIRCULATIONAHA.112.094524

Park KC, Gaze DC, Collinson PO, and Marber MS. 2017. Cardiac troponins: from myocardial infarction to chronic disease. Cardiovascular Research 113:1708-1718. $10.1093 / \mathrm{cvr} / \mathrm{cvx} 183$

Qipshidze Kelm N, Piell KM, Wang E, and Cole MP. 2018. MicroRNAs as predictive biomarkers for myocardial injury in aged mice following myocardial infarction. Journal of Cellular Physiology 233:5214-5221. 10.1002/jcp.26283

Sandoval Y, Smith SW, Sexter A, Thordsen SE, Bruen CA, Carlson MD, Dodd KW, Driver BE, $\mathrm{Hu}$ Y, Jacoby K, Johnson BK, Love SA, Moore JC, Schulz K, Scott NL, and Apple FS. 2017. Type 1 and 2 Myocardial Infarction and Myocardial Injury: Clinical Transition to High-Sensitivity Cardiac Troponin I. American Journal of Medicine 130:14311439.e1434. 10.1016/j.amjmed.2017.05.049

Smoot ME, Ono K, Ruscheinski J, Wang PL, and Ideker T. 2011. Cytoscape 2.8: new features for data integration and network visualization. Bioinformatics 27:431-432. 10.1093/bioinformatics/btq675

Su G, Morris JH, Demchak B, and Bader GD. 2014. Biological network exploration with Cytoscape 3. Curr Protoc Bioinformatics 47:8.13.11-24. 10.1002/0471250953.bi0813s47

Su Q, Liu Y, Lv XW, Ye ZL, Sun YH, Kong BH, and Qin ZB. 2019. Inhibition of lncRNA TUG1 upregulates miR-142-3p to ameliorate myocardial injury during ischemia and

PeerJ reviewing PDF | (2019:12:43763:1:1:NEW 13 Mar 2020) 
507

508

509

510

511

512

513

514

515

516

517

518

519

520

521

522

523

524

525

526

527

528

529

530

531

532

533

534

535

536

537

538

539

540

541

542

543

544

545

546

547

reperfusion via targeting HMGB1- and Rac1-induced autophagy. Journal of Molecular and Cellular Cardiology 133:12-25. 10.1016/j.yjmcc.2019.05.021

Tan NS, Goodman SG, Cantor WJ, Russo JJ, Borgundvaag B, Fitchett D, Dzavik V, Tan MK, Elbarouni B, Lavi S, Bagai A, Heffernan M, Ko DT, and Yan AT. 2016. Efficacy of Early Invasive Management After Fibrinolysis for ST-Segment Elevation Myocardial Infarction in Relation to Initial Troponin Status. Canadian Journal of Cardiology 32:1221 e1211-1221 e1218. 10.1016/j.cjca.2016.01.010

Vogel B, Claessen BE, Arnold SV, Chan D, Cohen DJ, Giannitsis E, Gibson CM, Goto S, Katus HA, Kerneis M, Kimura T, Kunadian V, Pinto DS, Shiomi H, Spertus JA, Steg PG, and Mehran R. 2019. ST-segment elevation myocardial infarction. Nat Rev Dis Primers 5:39. 10.1038/s41572-019-0090-3

Wang GK, Zhu JQ, Zhang JT, Li Q, Li Y, He J, Qin YW, and Jing Q. 2010. Circulating microRNA: a novel potential biomarker for early diagnosis of acute myocardial infarction in humans. European Heart Journal 31:659-666. 10.1093/eurheartj/ehq013

Wang X, Zhang Y, Wang H, Zhao G, and Fa X. 2017. MicroRNA-145 Aggravates HypoxiaInduced Injury by Targeting Rac1 in H9c2 Cells. Cellular Physiology and Biochemistry 43:1974-1986. 10.1159/000484121

Yan J, Yan JY, Wang YX, Ling YN, Song XD, Wang SY, Liu HQ, Liu QC, Zhang Y, Yang PZ, Wang XB, and Chen AH. 2019. Spermidine-enhanced autophagic flux improves cardiac dysfunction following myocardial infarction by targeting AMPK/mTOR signaling pathway. British Journal of Pharmacology. 10.1111/bph.14706

Yang H, Sun W, Quan N, Wang L, Chu D, Cates C, Liu Q, Zheng Y, and Li J. 2016. Cardioprotective actions of Notch1 against myocardial infarction via LKB1-dependent AMPK signaling pathway. Biochemical Pharmacology 108:47-57. 10.1016/j.bcp.2016.03.019

Yang Y, Cheng HW, Qiu Y, Dupee D, Noonan M, Lin YD, Fisch S, Unno K, Sereti KI, and Liao R. 2015. MicroRNA-34a Plays a Key Role in Cardiac Repair and Regeneration Following Myocardial Infarction. Circulation Research 117:450-459. 10.1161/CIRCRESAHA.117.305962

Yu D, Lim J, Wang X, Liang F, and Xiao G. 2017. Enhanced construction of gene regulatory networks using hub gene information. 18:186. 10.1186/s12859-017-1576-1

Zhao X, Wang K, Hu F, Qian C, Guan H, Feng K, Zhou Y, and Chen Z. 2015. MicroRNA-101 protects cardiac fibroblasts from hypoxia-induced apoptosis via inhibition of the TGFbeta signaling pathway. International Journal of Biochemistry and Cell Biology 65:155164. 10.1016/j.biocel.2015.06.005 
548

549

550

551

552

553

554

555

556

557

558

559

560

561

562

563

564

565

566

567

568

569

570

571

572

573

574

575

576

577

578

579

580

581

582

583

584

585

586

587

588

\section{Figure legends}

Figure 1. The identification of DE-miRNAs.

A, Volcano plot of DEGs in GSE24591. B, Volcano plot of DEGs in GSE31568. C, A Venn diagram between GSE24591 and GSE31568.

Figure 2. miRNA-target gene interactions.

Red dots represent miRNAs, and green dots represent target mRNAs.

Figure 3 Top 10 GO and KEGG terms of DEGs.

A, BP; B, CC; C, MF; D, KEGG.

Peer) reviewing PDF | (2019:12:43763:1:1:NEW 13 Mar 2020) 
589 Figure 4. The PPI of the top 100 DE-miRNAs.

590 Red indicates the 10 highest-degree genes, and the circles with green represent the remaining 591 genes. Edges are distinguished using colour shading from white to yellow.

592 Figure 5. Biological analysis of hub genes.

593 A, Interactions of 10 hub genes; B, KEGG enrichment analysis by Cytoscape; C, top 10 KEGG 594 enrichment pathways, as analysed in R.

595 Figure 6. Relative expression of DE-miRNAs in H9C2 cells.

596 A, miR-24-1*; B, miR-33a; C, miR-34a; D, miR-101; E, miR-139-3p; F, miR-142-3p; G, miR597 598. 'con' denotes the control H9C2 cell group, and 'DOX' denotes the group of H9C2 cells 598 treated with DOX $(2 \mu \mathrm{M})$.

599 Figure 7. ROC analysis of DEGs between AMI patients and control individuals.

600 A, miR-24-1*; B, miR-33a; C, miR-34a; D, miR-101; E, miR-139-3p; F, miR-142-3p; G, miR601598. 
Table $\mathbf{1}$ (on next page)

TABLE 1 The primer used in QPCR. 
TABLE 1 | The primer used in QPCR.

$\begin{array}{ll}\text { hsa-miR-545 } & \text { Forward: 5'-TCAGTAAATGTTTATTAGATGA-3' } \\ \text { hsa-miR-139-3p } & \text { Forward: 5'-GGAGACGCGGCCCTGTTGGAG-3' } \\ \text { hsa-miR-101 } & \text { Forward: 5'-CGGCGGTACAGTACTGTGATAA-3' } \\ \text { hsa-miR-24-1* } & \text { Forward: 5'-TGCCTACTGAGCTGATATCAGT-3' } \\ \text { hsa-miR-598 } & \text { Forward: 5'-CGTACGTCATCGTTGTCATCGTCA-3' } \\ \text { hsa-miR-33a } & \text { Forward: 5'-AGCGTGCATTGTAGTTGCATTGCA-3' } \\ \text { hsa-miR-142-3p } & \text { Forward: 5'-TGTAGTGTTTCCTACTTTATGGA -3' } \\ \text { hsa-miR-34a } & \text { Forward: 5'-CGCGTGGCAGTGTCTTAGCT-3' }\end{array}$

1 
Table 2 (on next page)

TABLE 2 The DE-miRNAs. 
1 TABLE $2 \mid$ The DE-miRNAs.

\begin{tabular}{llll}
\hline Symbol & P Value & $\log F C$ & Up/Down \\
\hline hsa-miR-545 & 0.00038 & -1.50467 & Down \\
hsa-miR-139-3p & 0.0005900000 & -1.53651 & Down \\
hsa-miR-101 & 0.0080000000 & 1.04767 & Up \\
hsa-miR-24-1* & 0.0001180000 & -2.08228 & Down \\
hsa-miR-598 & 0.0036000000 & -1.14074 & Down \\
hsa-miR-33a & 0.0002760000 & -1.98301 & Down \\
hsa-miR-142-3p & 0.0002622 & -1.07909 & DOWN \\
hsa-miR-34a & 0.0031100000 & 1.90204 & Up \\
\hline
\end{tabular}

2 
Table 3 (on next page)

Table 3 The miRNA-mRNA network. 
TABLE 3 | The miRNA-mRNA network.

\begin{tabular}{|c|c|c|c|}
\hline Symbol & Up/Down & Count & Target mRNA \\
\hline hsa-miR-545 & Down & 85 & $\begin{array}{l}\text { USP15, WEE1, DDX3X, CSNK1G3, LRP1, FGF2, CRIM1, FAM13A, ZFHX4, } \\
\text { PEX5L, RAB10, AGO4, PLAG1, MAP3K7, HIGD1A, ZCCHC3, CNOT6L, } \\
\text { PLEKHA1, PIP4K2C, NLK, ARID2, PSAT1, RNF138, PTMA, BNC2, FZD6, } \\
\text { SLC25A12, SYPL1, COL12A1, LRIG1, NUP50, ARIH1, RNF111, CDC42SE2, } \\
\text { TRIM37, GPATCH8, ZFR, PURB, ARHGAP12, SNAI2, PEG10, CCND1, YWHAQ, } \\
\text { WNK3, PRDM4, HMGB3, FXR1, PLAGL2, SMAD7, ACVR2B, CDV3, RREB1, } \\
\text { DIP2C, GABARAPL1, UBN2, CDCA4, MTDH, ATG14, TM9SF3, SLC7A2, } \\
\text { HNRNPDL, DCAF17, SLC16A9, SPRED1, TMEM33, ZFHX3, ZNF704, VCAN, } \\
\text { NDUFB6, MID1, PARM1, CNTLN, MKX, SMG1, PHF3, IGF2R, ZNF622, PODXL, } \\
\text { PPM1A, NEGR1, ATP13A3, ACBD5, STRBP, EN2, ZFP91 }\end{array}$ \\
\hline hsa-miR-139-3p & Down & 12 & $\begin{array}{l}\text { MTDH, SOX4, ZBTB7A, EHD3, ALOX15, ZNF585B, FAM162A, ZNF589, TBX20, } \\
\text { HLA-A, FOXC1, MRPL9 }\end{array}$ \\
\hline hsa-miR-101 & Up & 205 & $\begin{array}{l}\text { DUSP1, EZH2, FBN2, ATXN1, ARID1A, RAP1B, MYCN, TGFBR1, AEBP2, } \\
\text { BICD2, FOS, BCL9, MBNL1, RAB5A, ANKRD17, ZNF207, RANBP9, RAP2C, } \\
\text { MOB4, NLK, DNMT3A, ZCCHC2, FNDC3A, NACA, PTGS2, TNPO1, } \\
\text { PAFAH1B1, MITF, RNF111, CBFA2T2, SMARCD1, ZBTB18, MAP3K4, SOX9, } \\
\text { DYRK2, SMARCA5, LCOR, ZNF654, LMNB1, SUB1, HNRNPF, UBE2D3, ICK, } \\
\text { MBNL2, SIX4, OTUD4, INO80D, ZEB2, APP, ABHD17C, MRGBP, ARID5B, } \\
\text { CADM1, RREB1, MET, CDH5, STMN1, MFSD6, TSPAN12, TMEM161B, TET2, } \\
\text { PURB, SYNCRIP, PPP2R2A, UBE2A, ZEB1, AP1G1, NR2F2, PPP2R5E, FMR1, } \\
\text { TGIF2, ZFP36L2, ANKRD11, LIFR, PHF3, CERS2, NEK7, MPPE1, ZFX, } \\
\text { PRKAA1, TNRC6B, GNB1, BZW1, TMED5, UBN2, CPEB1, DDIT4, FZD6, } \\
\text { FBXW7, KLF12, LRCH2, ZNF451, EED, HNRNPAB, PIP5K1C, RORA, EIF4G2, } \\
\text { SLC38A2, ATXN1L, RNF219, C1orf52, BCL2L11, NAP1L1, C8orf4, KDM6B, } \\
\text { ZC3H11A, DIDO1, ZBTB21, KDM3B, MLEC, STAMBP, MTSS1L, ARAP2, } \\
\text { POU2F1, ACVR2B, BEND4, PIK3C2B, NUFIP2, FAM84B, C10orf88, SPATA2, } \\
\text { NUPL2, MAML3, PSPC1, SGPL1, KLF6, LRRC1, RAC1, TMEM170B, RAB39B, } \\
\text { TMEM68, LBR, PLEKHA1, AKAP11, HSPA13, MCL1, AFF4, SACM1L, ZNF800, } \\
\text { AP1S3, CAPN2, FRS2, SREK1IP1, MRPL42, FAR1, TRERF1, RNF213, WWTR1, } \\
\text { NACA2, SLC39A6, WNK1, TFAP4, DAZAP2, CNEP1R1, CBX4, SSFA2, SPIRE1, } \\
\text { GOLGA7, ATG4D, MORC3, TGFBR3, SNRNP27, ADO, TGOLN2, LIN7C, MNX1, } \\
\text { PANK1, GPAM, MTOR, NAA30, TMTC3, TBC1D12, PRPF38B, BLOC1S6, } \\
\text { ELAVL2, KIAA1586, TBX18, DENND5B, TNFAIP1, KPNA2, NXT2, RAB11FIP1, } \\
\text { N4BP1, PEX5L, CLIC4, VEZT, NACC1, AP3M1, FBXO11, E2F3, TEAD1, } \\
\text { CDKN1A, ZNF350, PLAG1, ZNF645, REL, CMTM6, STX16, XPO7, CHAMP1, } \\
\text { RNF44, DIMT1, QSER1, FAM217B, ATP5B }\end{array}$ \\
\hline
\end{tabular}

hsa-miR-24-1 Down $25 \quad$ SLITRK1, FOXM1, CYP20A1, DPCR1, CLNS1A, RACGAP1, ZWINT, ELOVL5, DHX33, PRMT7, PRKAR1A, ZNF367, TVP23C, DDIT4, TPM4, SLC23A3, SKAP2, 
hsa-miR-33a

Down

93

hsa-miR-142-3p Up

hsa-miR-34a Up
KPNA4, ZNF281, NPC1, ABCA1, YWHAH, HMGA2, CROT, ARID5B, PIM1, ABHD2, IRS2, SLC26A7, RMND5A, GAS1, PAPOLG, SGCD, STYX, ZC3H12C, CPEB2, ANKRD29, BTBD2, FAM46C, NUFIP2, PTHLH, RAB5A, DSC3, PIM3, FOXP1, SLC16A1, PPARA, TSG101, PCM1, LDHA, ZCCHC14, TWIST1, PDE8A, SP1, FCHO2, REEP1, SOCS5, RBM33, SEMA7A, STRBP, HBS1L, PPP1R9A, PGM3, UBE2D1, SRSF1, MLLT10, RNF19A, LIMA1, XIAP, GDNF, SLC39A14, NCOA3, SAMD8, CREBBP, NFYC, RAP2C, SEC62, SNTB2, SECISBP2L, SPIN4, TCF12, HIF1A, GIGYF1, CNOT4, MAPK8, TIA1, PDIA6, FIGN, KLHL15, ITGBL1, BCL10, PLEKHA8, DCBLD2, ACSL4, TMEM65, EDN1, FBXO30, ELL2, G2E3, ZNF208, RARB, CAPRIN1, MATR3, PDE10A, BRWD1, IGF1R, KLF9, HSPA13, WNT5A, CEP170

11 CLTA, ZNF217, HMGA2, CPEB2, ZCCHC14, SGMS1, ACSL4, ZFX, XIAP, ZFP36L2, KLHL15

159 SYT1, NOTCH1, DLL1, PDGFRA, SATB2, E2F5, FUT8, LEF1, TPD52, FOXP1, UBP1, E2F3, JAG1, POGZ, MET, GALNT7, FOXN3, ZNF644, ACSL1, BCL2, NR4A2, VAMP2, ACSL4, SGPP1, MYCN, RRAS, PEA15, KLF4, CCNE2, MAP2K1, AXL, EVI5L, SAR1A, CAMTA1, YY1, SIRT1, LDHA, TMEM109, SLC4A7, BAZ2A, MTA2, CSF1R, FOSL1, ARHGAP1, GMFB, BMP3, INHBB, CCND1, MTMR9, NOTCH2, GAS1, PODXL, SNTB2, VPS37B, MDM4, ZDHHC16, PPP1R16B, GRM7, CPEB3, CDK6, IL6R, NCOA1, HSPA1B, TSN, SURF4, FAM46A, RDH11, LRRC40, CNOT4, VCL, PPP1R10, METAP1, PEG10, HOXA13, EFNB1, STX1A, ADO, STRAP, CLOCK, LMAN1, SMAD4, SOCS4, AREG, PLCG1, DOCK3, SLC35G2, POU2F1, PHF19, TM9SF3, CCL22, HNF4A, RAE1, EDEM3, MYB, CDC25A, TNRC18, PPP3R1, TPPP, SHOC2, TOM1, WNT1, BCL2L13, IGF2BP3, MOAP1, GORASP2, MYO1C, SRC, KIT, KMT2D, CBX3, UBL4A, GIGYF1, CYB5B, MBD6, HDAC1, SNAI1, ZNF551, SSX5, ARHGDIB, ERLIN1, FIGN, TSPAN14, ZC3H4, ULBP2, ATP13A3, CDKN1B, STAG2, NEUROD2, AXIN2, RTN4, RRAGD, FAM208A, MAZ, ATXN2L, ABLIM1, IGSF3, UST, CDAN1, JARID2, WDR77, LRRFIP1, SNX12, CTIF, NDRG1, TMOD2, UCK2, HNRNPU, BIRC5, MTAP, RBM12, TMEM167A, XIAP, SLC4A2, AK2, EFHD2, RNF169, IRGQ, DDX10, L1CAM 


\section{Table 4 (on next page)}

TABLE 4 Top 10 genes in network ranked by Degree method. 
1 TABLE 4 | Top 10 genes in network ranked by Degree method.

\begin{tabular}{lll}
\hline Rank & Symbol & Score \\
\hline 1 & CTNNB1 & 50 \\
2 & NOTCH1 & 47 \\
3 & CCND1 & 41 \\
4 & EZH2 & 36 \\
5 & MTOR & 33 \\
6 & CDKN1B & 31 \\
7 & CDKN1A & 29 \\
8 & MAP2K1 & 27 \\
9 & BTRC & 26 \\
10 & RAC1 & 24 \\
\hline
\end{tabular}




\section{Figure 1}

Figure1 Identification of differentially expressed miRNAs analysis

Figure $1 \mathrm{~A}$, Volcano plot of differentially expressed miRNAs in GSE 24591. The red dot represents upregulated miRNAs and the green dot represents downregulated miRNAs. B, Volcano plot of differentially expressed miRNAs in GSE31568. The red dot represents upregulated miRNAs and green dot represents downregulated miRNAs. miRNAs, microRNAs. C, A Venn-diagram between GSE 24591 and GSE 31568. The coincident part represents the differentially expressed genes shared by the two series, accounting for a total of 8 .

A

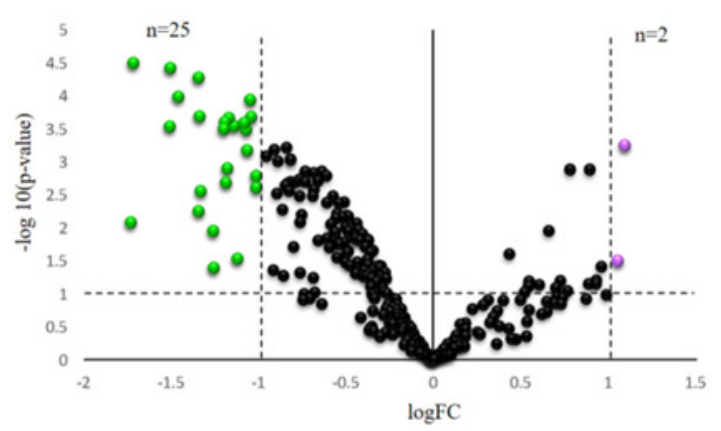

C

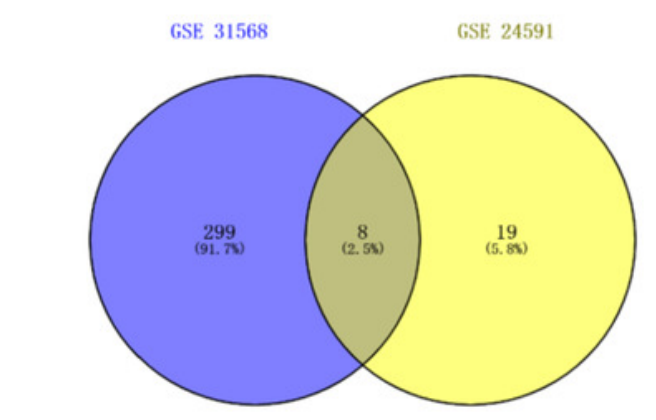

B

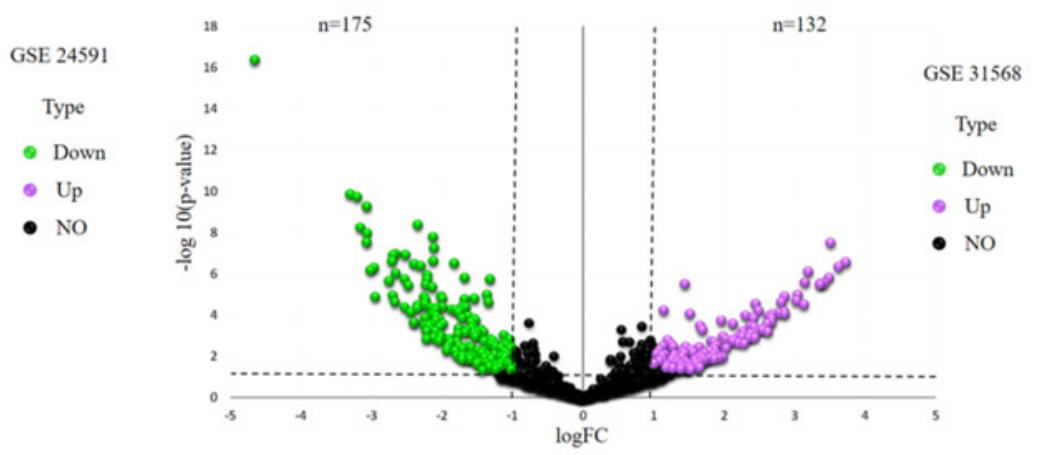


Figure 2

Figure2 MiRNA-target gene interactions

Interaction networks of miRNA and target DEGs in AMI. The red dot represents miRNAs and the green dot represents target mRNAs.

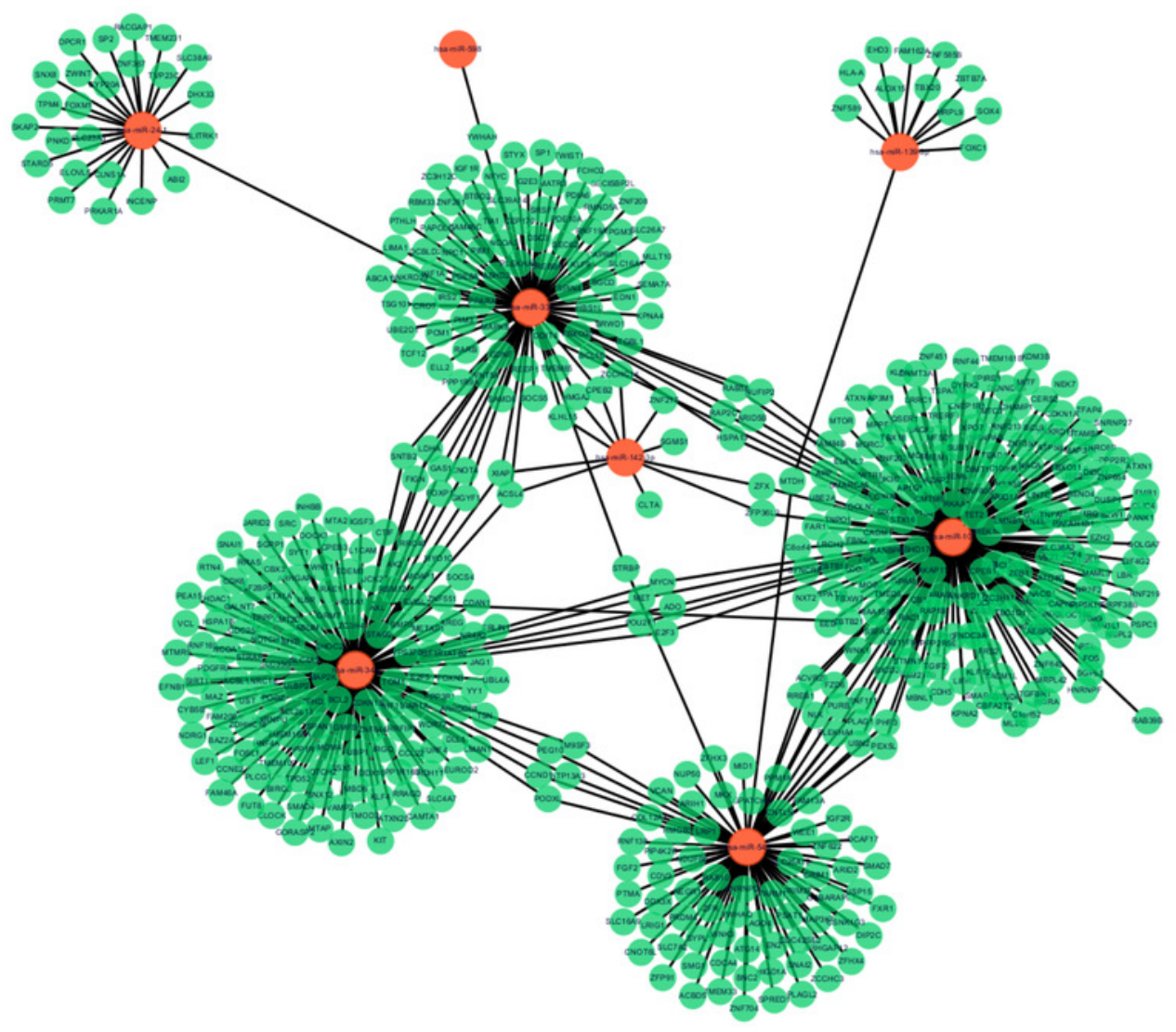


Figure 3

Figure3 Top 10 significant enrichment GO and KEGG terms of DEGs

Figure 3A, BP: biological process; B, CC: cellular component; C,MF: molecular function; D, KEGG: signaling pathway.

$\mathbf{A}$

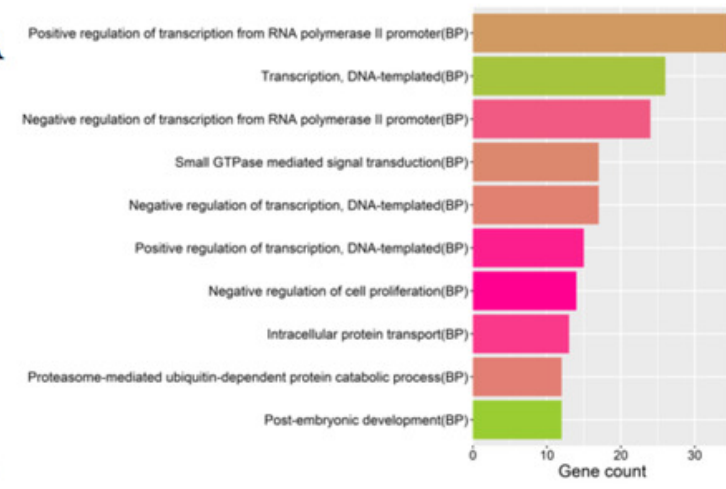

C

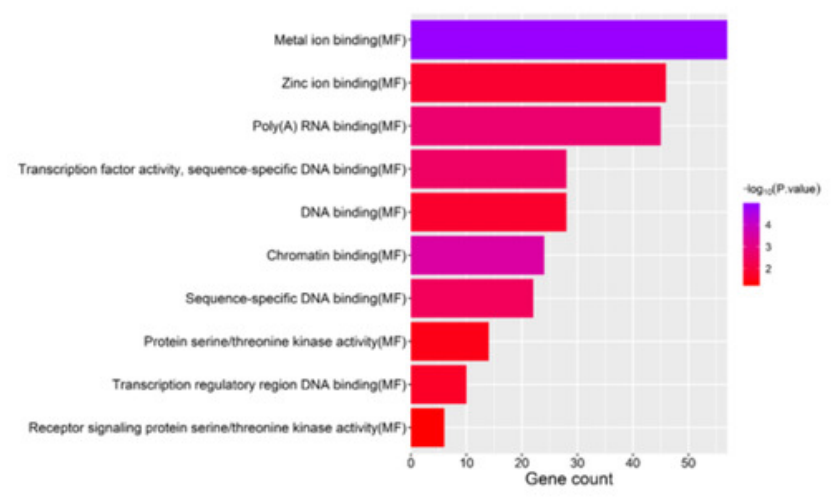

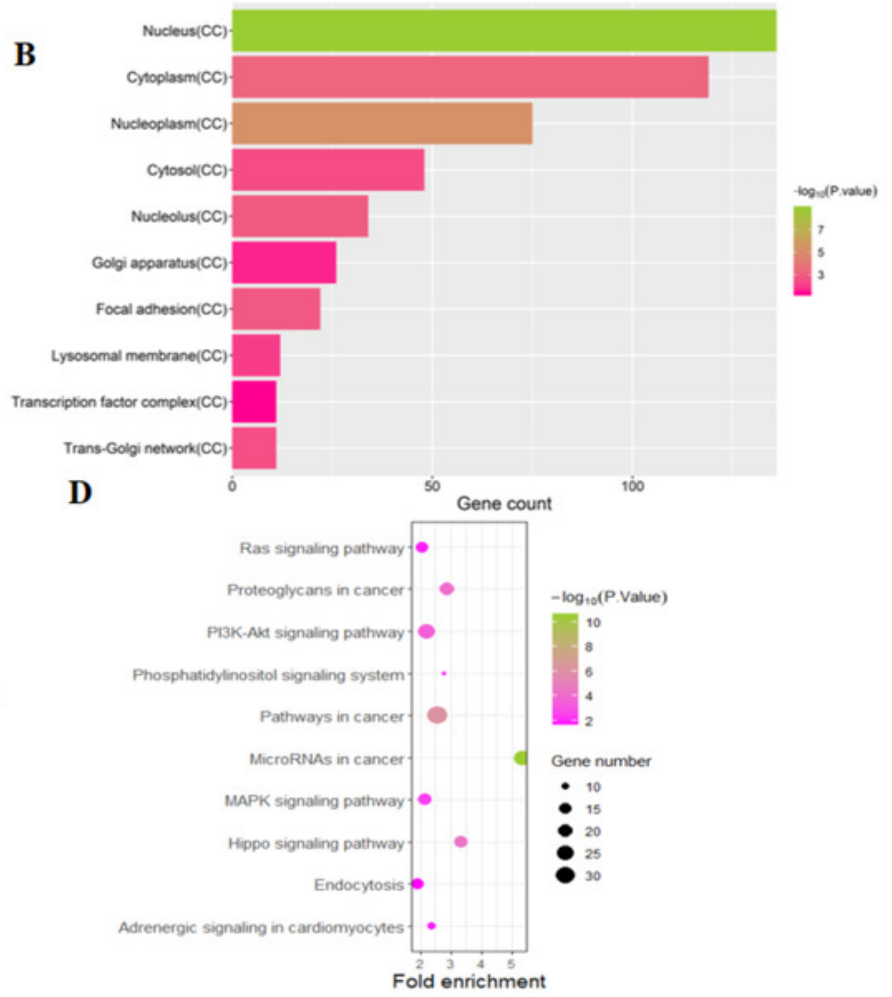


Figure 4

Figure4 The PPI networks of top 100 DEGs

All the circles are proteins encoded by top 100 DEGs. The red colors represent the 10 highest degree genes and the circles with green represent the remaining genes. Edges are distinguished using the color shading from white to yellow.

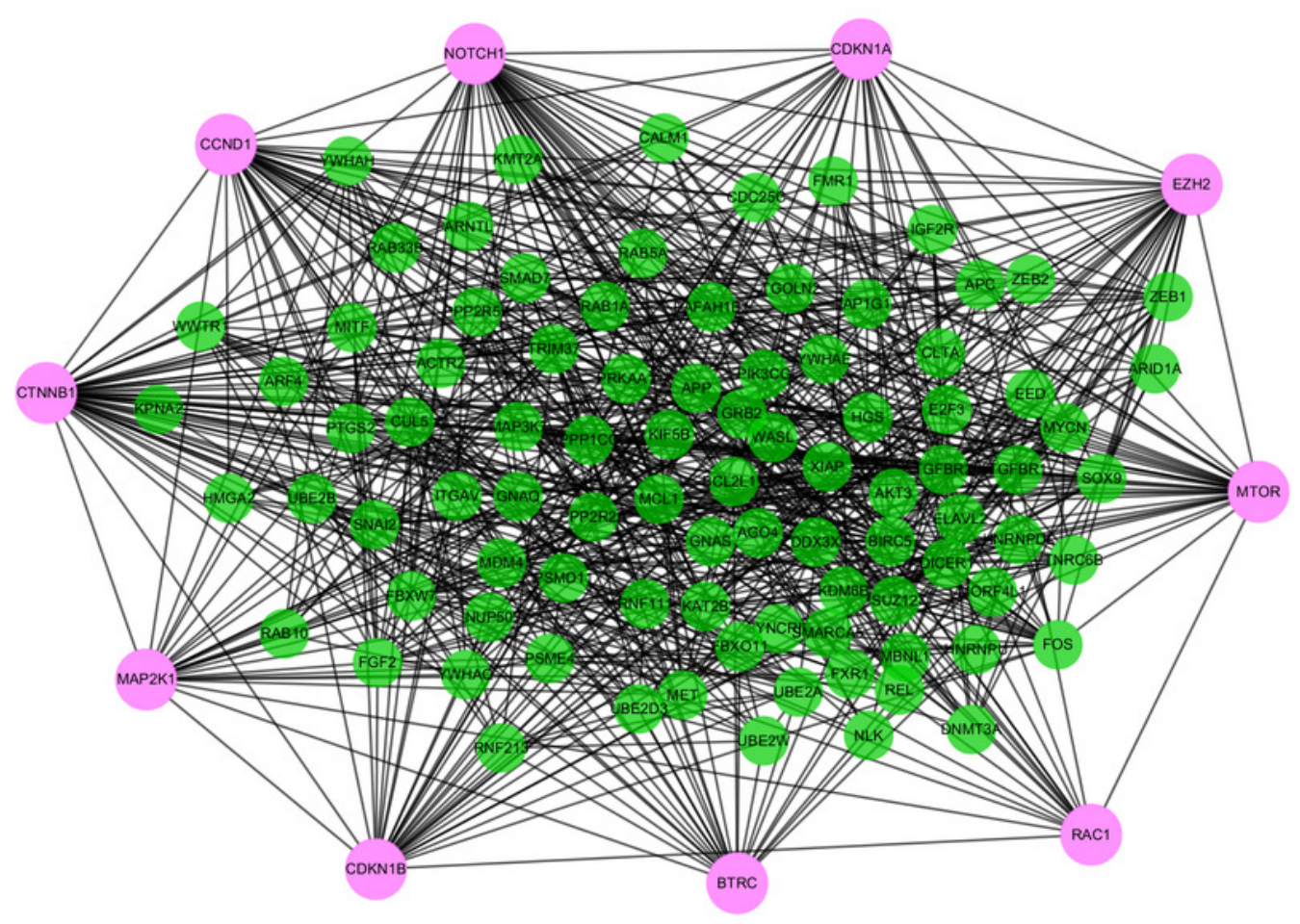


Figure 5

Figure5 Biological analysis of hub genes

Figure 5A, the interaction of 10 hub genes; B, the KEGG enrichment analysis by Cytoscape; C, the top 10 KEGG enrichment analysis by $R$ language.

A

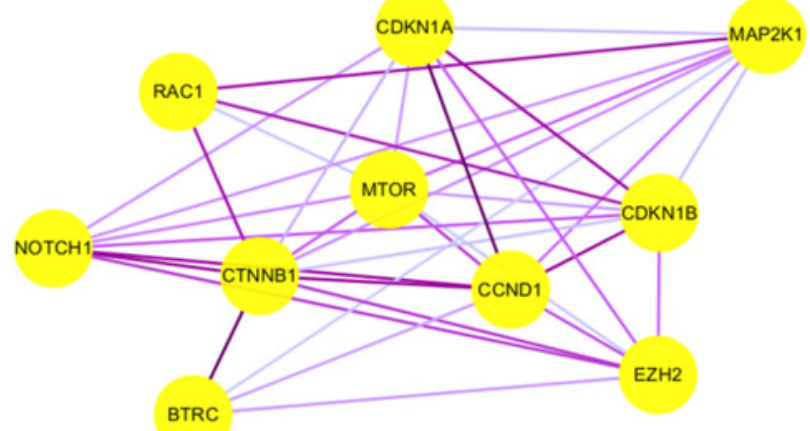

C

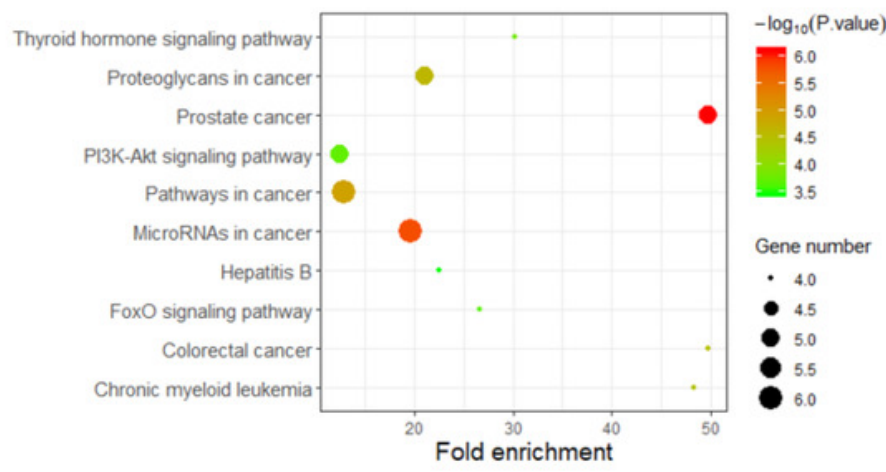

B

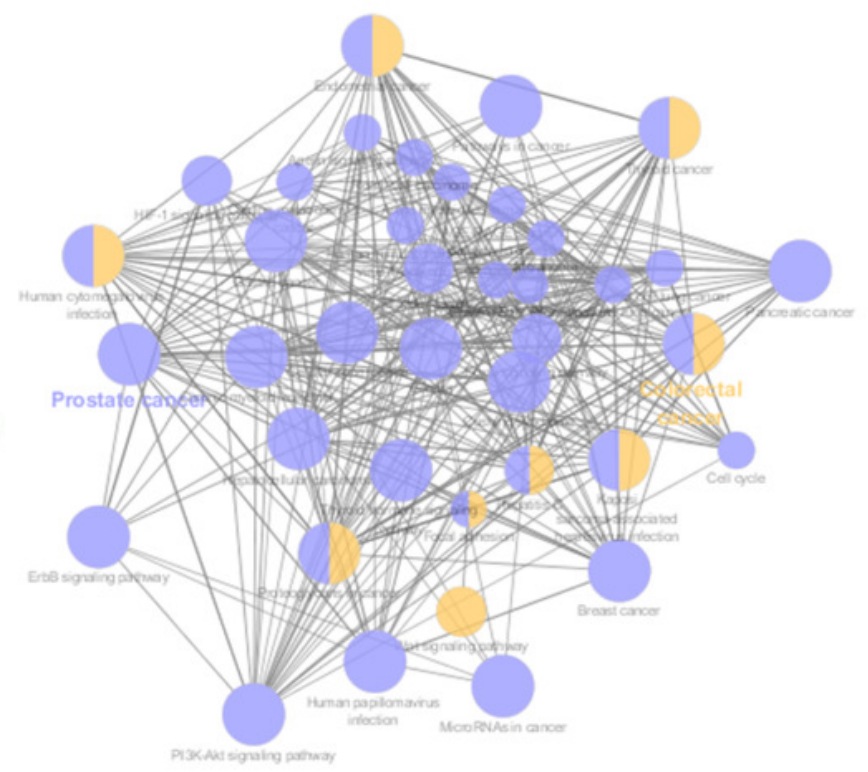


Figure 6

Figure6 The relative expression of differentially expressed miRNA in $\mathrm{H} 9 \mathrm{C} 2$.

Figure6A, miR-24-1*; B, miR-33a; C, miR-34a; D, miR-101; E, miR-139-3p; F, miR-142-3p; G, miR-545; H, miR-598. The group of con respects normal H9C2 and the group of DOX respect the cell of $\mathrm{H} 9 \mathrm{C} 2$ treated with DOX $(2 \mu \mathrm{M})$.

A

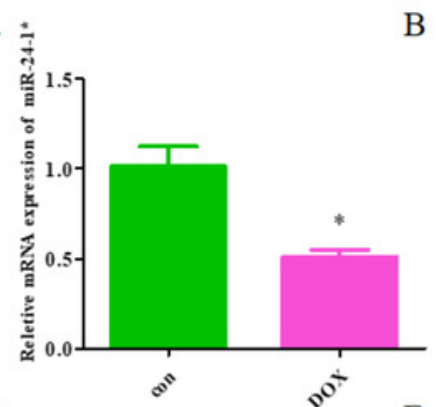

$\mathrm{E}$

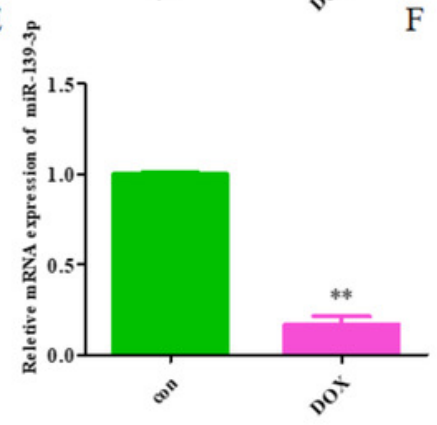

B
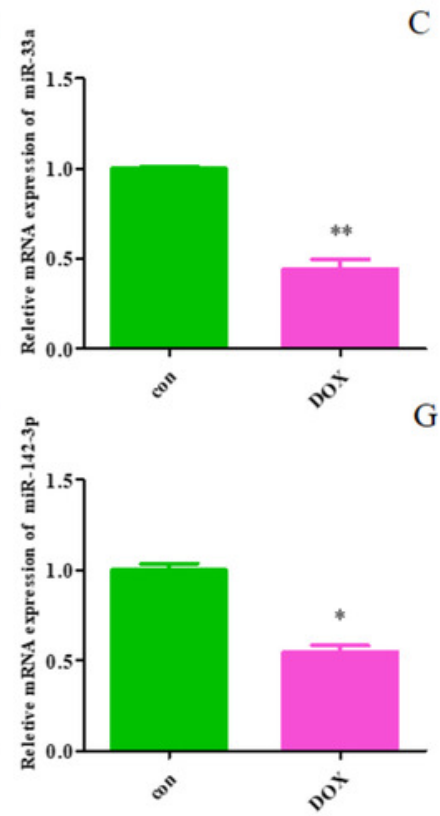

$\mathrm{C}$
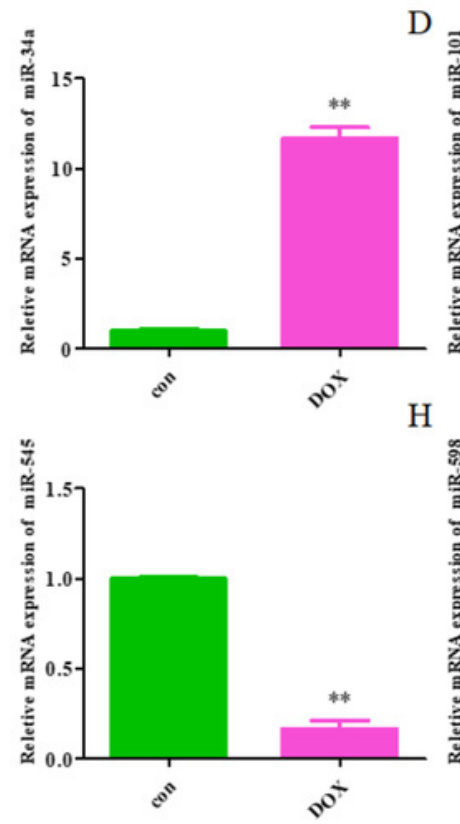
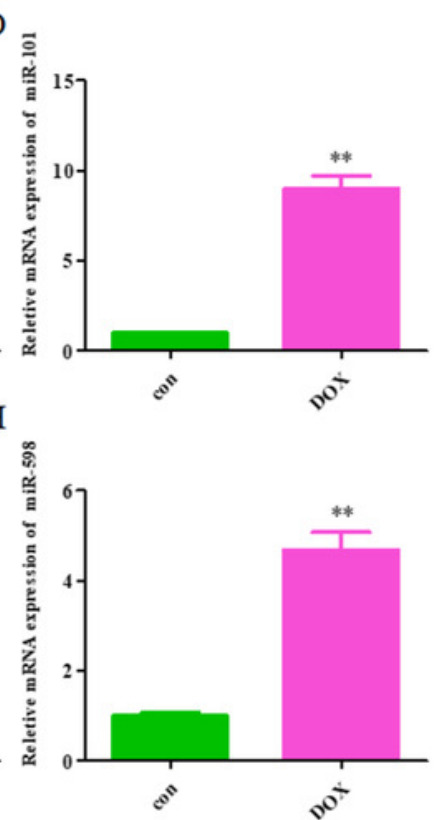
Figure 7

Figure7 Receiver operating characteristic curves (ROC) of differentially expressed miRNA between AMI patients and healthy controls.

Figure7A, miR-24-1*; B, miR-33a; C, miR-34a; D, miR-101; E, miR-139-3p; F, miR-142-3p; G, miR-545; H, miR-598

A

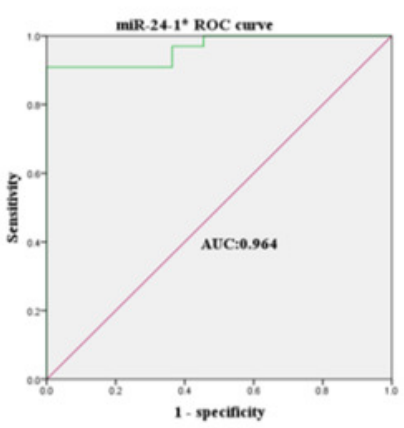

E

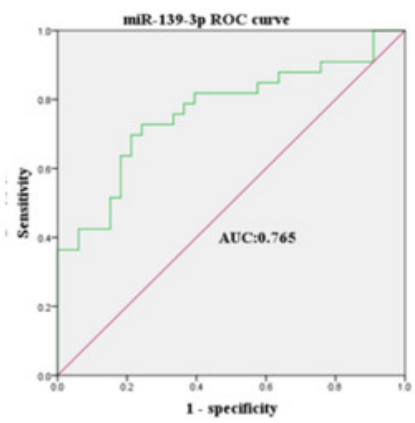

B

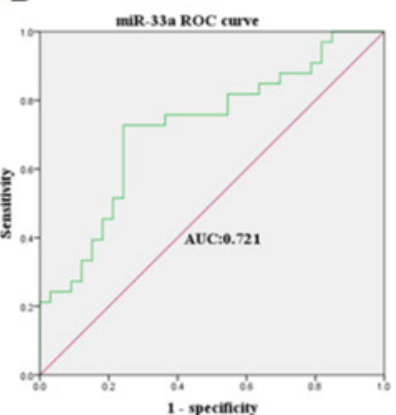

$\mathrm{F}$

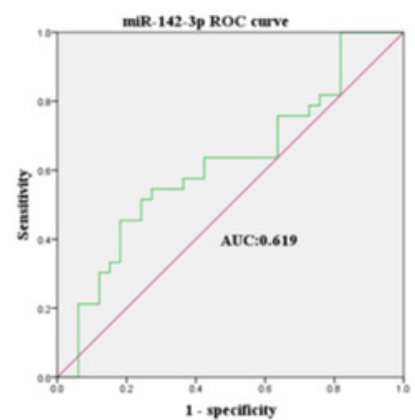

C

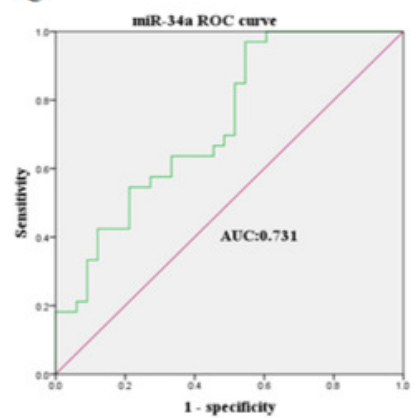

G

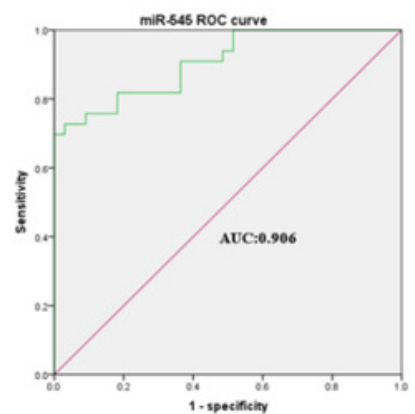

D

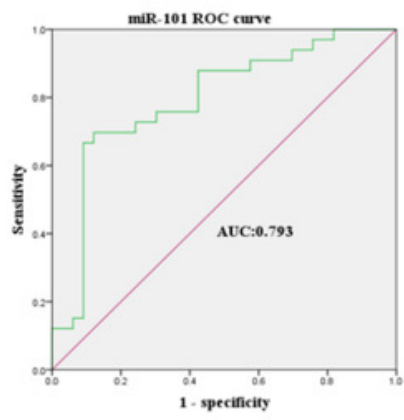

$\mathrm{H}$

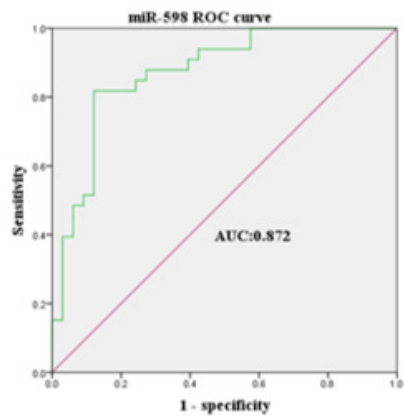

